Trauma Berufskrankh 2016 - [Suppl 4]:

18:S353-S358

DOI 10.1007/s10039-016-0146-5

Online publiziert: 10. Mai 2016

๑) Springer-Verlag Berlin Heidelberg 2016

CrossMark

Hartmut Semsch

OMM ORTEMA GmbH, Markgröningen, Deutschland

\title{
Versorgungsmöglichkeiten der oberen Extremität aus Sicht des Orthopädie-Technikers
}

Ein weiterer Punkt ist die Spezialisierung der Fachärzte und die dadurch bedingte Aufteilung in verschiedene regionale Schwerpunkte, sodass Schulter, Ellbogen und Hand oftmals von unterschiedlichen Spezialisten behandelt werden. Große orthopädische und handchirurgische Zentren sind nicht überall vorhanden, wodurch sich die Fallzahlen nochmals dividieren. Auch fehlen gute Klassifikationen der Verletzungen und entsprechend klar vorgegebene Richtlinien zur Therapie und technischen Versorgung. Es be- stehen keine Standards, welche Verletzungen konservativ behandelt gut ausheilen, welche operativ stabilisiert und korrigiert werden müssen oder welche in einer chronischen Instabilität münden können. Traumatische Verletzungen stehen auch oft in Zusammenhang mit Frakturen, hier steht allgemein die Bewegungslimitierung zur Schmerzreduktion im Vordergrund (• Abb. 1).

Orthesen werden auch heute noch häufig als Anschlussversorgung an eine Gipsruhigstellung gesehen, wobei seit
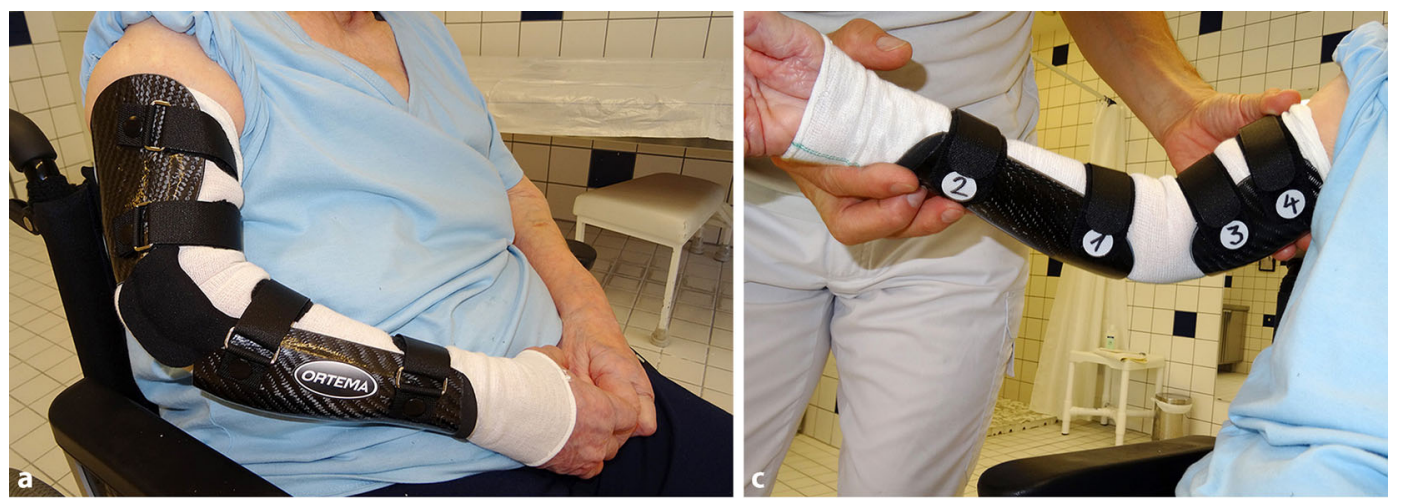

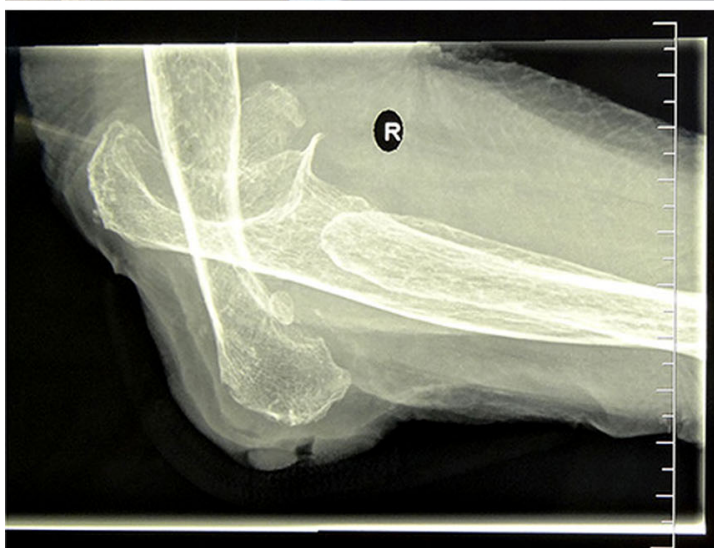

$10 \mathrm{~cm}$

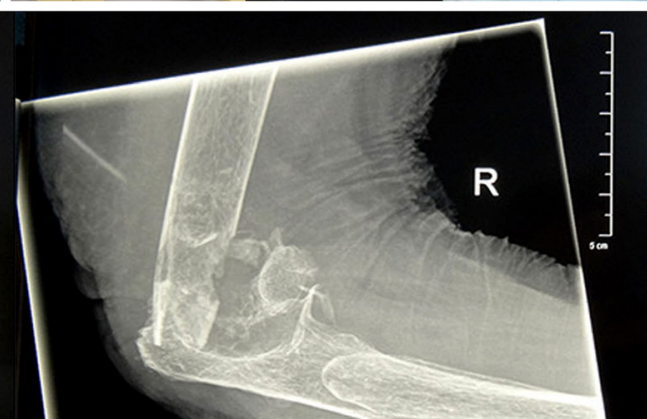

lat.
Abb. $1<$ a Technische Versorgung mit einer bewegungslimitierten Orthese. b Röntgenbild posttraumatisch, nach Reposition. c Monolaterale Gelenkführung mit Anziehhilfe 


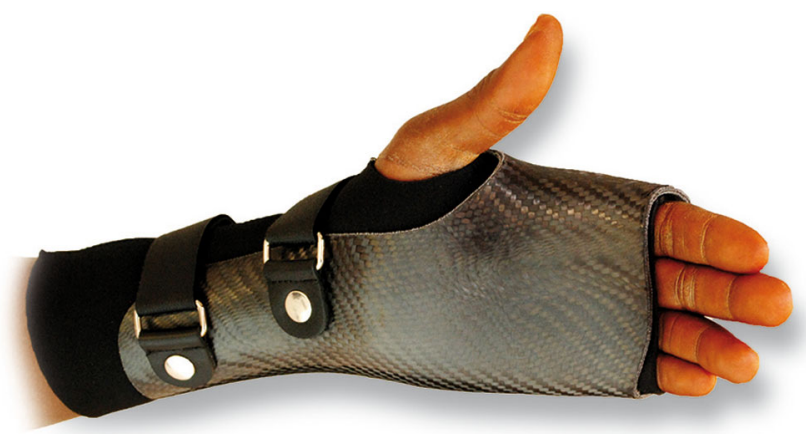

Abb. $2 \Delta$ Handversorgung nach Mittelhandfraktur eines Fußballspielers für den Spieleinsatz

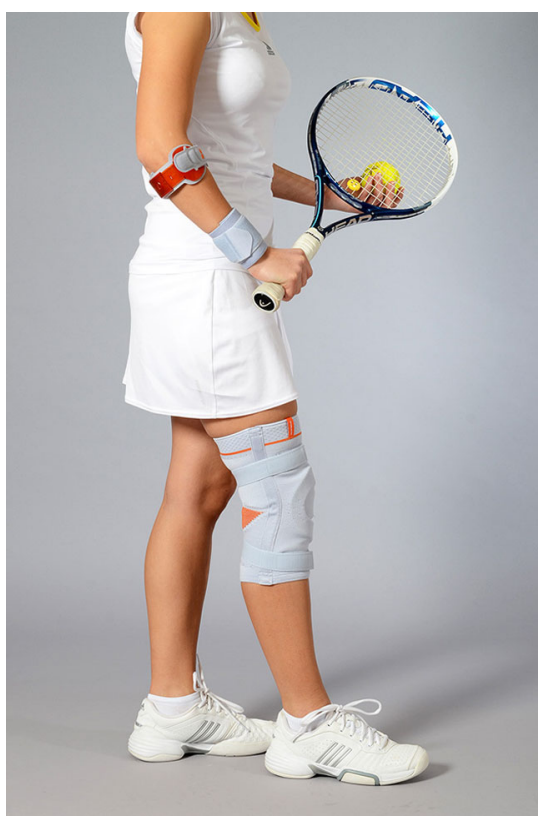

Abb. 4 ム Epikondylitisspange zur Entlastung der Sehnenansätze beim Tennis

Jahren erkannt wurde, dass mit der orthetischen Versorgung eine wesentlich bessere Beübung durch den Physiotherapeuten und schnellere Freigabe einer evtl. Gelenklimitierung gewährleistet ist.

Frakturen traumatischen Ursprungs werden häufig beim Sport erlitten. Hier steht im Leistungssport die Versorgung mit einer individuell nach Gipsabdruck gefertigten Kunststoff- oder Carbonorthese zur temporären Stabilisierung [2] und Ruhigstellung im Vordergrund. Sollten Frakturen im Bereich der Hand auftreten, gilt es auch weiterhin, die benachbarten Gelenke ruhig zu stellen (• Abb. 2), um im Zeitraum von 5 bis 6 Wochen ein gutes Behandlungsergebnis erreichen zu können.

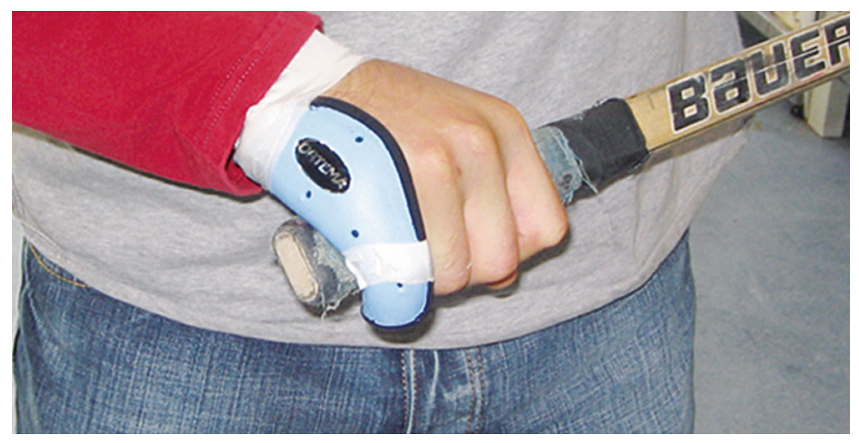

Abb. $3 \Delta$ Eishockeyspieler mit Orthesenversorgung in sportartspezifischer Stellung

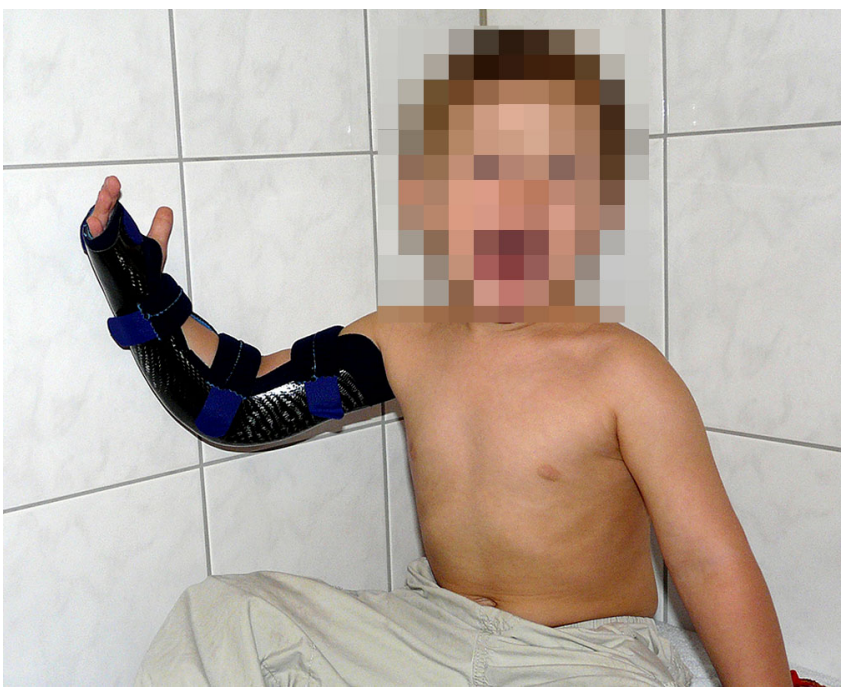

Meist luxiert ein Gelenk beim gesunden Menschen infolge eines Sturzes oder einer Sportverletzung, Häufigste Ursache der Ellenbogenluxation ist der Sturz auf den ausgestreckten Arm [5], der dabei unter axialer Lasteinleitung flektiert oder hyperextendiert. Hier kann es ebenfalls zur Luxationsfraktur bzw. zu ligamentären Verletzungen kommen. Die plötzliche Krafteinwirkung wird auf das Gelenk übertragen, und die Gelenkflächen verschieben sich.

Bei der Schulterluxation sind häufig muskuläre oder ligamentäre Zusatzverletzungen sowie Reluxationen oder schwere Rasanztraumen von großer Bedeutung für die technische Versorgung.

Kommt es an der oberen Extremität zu Ausfällen durch Plexusparesen, sind unter Umständen Schulter, Ellenbogen und Handgelenk orthetisch zu versorgen, was einen hohen fachlichen Anspruch an die Technik stellt.
Abb. $5<$ Nicht dislozierte Ellenbogenorthese bei einem 4-Jährigen nach Sturz vom Motorrad des Vaters. Individuell gefertigte Ellenbogenorthese in sog. .,Spuckhandstellung". Dadurch ist eine gute therapeutische Beübung aus der Orthese möglich

\section{Methodik}

In den letzten Jahren konnten umfangreiche Erfahrungen v. a. bei komplexen Rasanztraumen und Sportverletzungen gesammelt werden, die technisch jedoch indikationsabhängig unterschiedlich $\mathrm{zu}$ versorgen sind. Finger-, Handgelenkund Unterarmfrakturen werden in der Regel auch bei Sportlern in einer Funktionsstellung und unter Umständen auch in Abstimmung auf ein Sportgerät durchgeführt (• Abb. 3).

Ansonsten ist eine axial ausgerichtete Fixierung in der Intrinsic-plusStellung das Mittel der Wahl [6]. Dabei steht das Handgelenk in $20-30^{\circ}$ Streckung, die Fingergrundgelenke stehen in $70-80^{\circ}$ Beugung, die Finger-, Mittelund Endgelenke in $0-10^{\circ}$ Beugung. Der Daumen wird in mittlerer Oppositionsstellung gehalten. In dieser Stellung sind die Kollateralbänder straff und können nicht schrumpfen, eine Beugekontraktur 
der proximalen Interphalangealgelenke (PIP-Gelenke) wird dabei verhindert.

Verletzungen am Ellenbogen können akut traumatisch oder chronisch rezidivierend sein [1] und bieten daher ein vielseitiges Krankheitsbild. Akute Verletzungen betreffen Spitzenathleten gleichermaßen wie Hobbysportler, Überlastungserscheinungen stehen in einem engen Zusammenhang mit der spezifischen Beanspruchung des Ellenbogengelenkes. Kommt es zu Penetration, ist eine Reizung des Strecksehnenansatzes am radialen Epikondylus eine Folge, die zu lokalen Beschwerden führen kann. Mittels einer Epikondylitisspange können hier häufig Beschwerden effektiv behandelt werden (•Abb. 4).

Akute Verletzungen am Ellenbogen in Form von Frakturen oder Ellenbogenluxationen, können mit ossären und/ oder ligamentären Instabilitäten verbunden sein. Ein indirekter Kraftfluss infolge eines Sturzes auf den ausgestreckten Arm geht mit Radiuskopffrakturen oder Ellenbogenluxationen einher, wohingegen das direkte Anpralltrauma typisch ist für Olekranonfrakturen (• Abb. 5) [4].

Bei der Ellenbogenluxation wird generell entschieden, ob es sich bei einer technischen Versorgung um eine einseitige Gelenkführung und bilateral ausgeführte Konstruktion handelt. Diese sollte möglichst wenig auftragen und eng am Körper anliegen, jedoch darf die Flexion des Ellenbogens nicht beeinträchtigt werden. Eine Limitierung der Gelenkbewegung im Sinne eines Extentions-/ Flexionsstopps ist sinnvoll und wird vom behandelnden Arzt vorgegeben (• Abb. 6).

Sollte eine zusätzliche Fraktur vorliegen, kann mit einem zweiten, von frontal aufliegenden Schalenanteil eine zusätzliche Stabilisierung in der Art eines Fracture-Brace durch die zirkuläre Fassung erreicht werden. Um ausreichende Stabilität zu gewährleisten, sollten die Unterarm- sowie die Oberarmschale ca. zwei Drittel des jeweiligen Armanteils fassen, die Verschlusstechnik der Orthese wird praktischerweise über ausreichend breite Klettverschlüsse gewährleistet, welche die Ellenbogenorthese rutschfrei und effektiv am Arm fixieren.

Trauma Berufskrankh 2016 • [Suppl 4]: 18:S353-S358 DOI 10.1007/s10039-016-0146-5

(c) Springer-Verlag Berlin Heidelberg 2016

\section{H. Semsch \\ Versorgungsmöglichkeiten der oberen Extremität aus Sicht des Orthopädie-Technikers}

\section{Zusammenfassung}

Verletzungen an der oberen Extremität können akut traumatisch oder chronisch rezidivierend bedingt sein. Häufig sind Sportler betroffen, die bei der Ausübung ihrer Sportart Frakturen, Luxationen, aber auch Paresen erleiden. Bei Frakturen werden bei Aktiven regelmäßig Orthesen zur temporären Ruhigstellung gefertigt. Die Luxation eines Gelenkes tritt meist infolge eines Sturzes oder eines Traumas auf. Ungefähr $50 \%$ aller Luxationen betreffen dabei das Schultergelenk, gefolgt von der Ellenbogenluxation.
Dabei ist die technische Versorgung durch die große Beweglichkeit anspruchsvoll. Aufgrund der guten Ergebnisse kommen zunehmend Patienten mit Plexusparese und dem Wunsch nach Funktionswiederherstellung mittels einer Armorthese. Hierfür konnte eine hydraulische Ellenbogenunterstützung entwickelt werden.

Schlüsselwörter

Fraktur - Luxation · Parese · Sportler · Orthesen

\section{Treatment options for the upper extremities from the perspective of orthopedic technicians}

\section{Abstract}

Injuries to the upper extremities can be caused by acute trauma or can be a chronic recurrence. When practicing their form of sport athletes are often affected by fractures and dislocations as well as also suffering from palsy. Orthotic devices are regularly used for temporary immobilization of fractures in active athletes. The dislocation of a joint mostly occurs as a result of a fall or trauma. Approximately $50 \%$ of dislocations affect the shoulder joint followed by the elbow joint. In these cases the technical treatment is demanding due to the great range of movement. Because of the good results patients with brachial plexus palsy are coming increasingly more with the wish for restoration of function using an arm orthosis. A hydraulic elbow support has been developed for this purpose.

\section{Keywords}

Fracture $\cdot$ Dislocation $\cdot$ Palsy $\cdot$ Athletes . Orthotic devices
Diese Konstruktion kann auch bei einer Pseudoarthrose eingesetzt werden (• Abb. 7).

Die größte Schwierigkeit besteht in der Festlegung des mechanischen Gelenkdrehpunktes. Hier macht es Sinn, beim Gipsabdruck auf eine exakte Anzeichnung der Epikondylen zu achten, um anschließend bei der Festlegung des Drehpunktes eine gute Orientierung zu haben.

Bei der orthetischen Versorgung der Schulter müssen mit dem behandelnden Arzt die Art und Weise der Stabilisierung und die zulässige Gelenkbewegung im Vorfeld abgesprochen werden. Informationen über die Verletzungsart wie Rotatorenmanschettenruptur, Schultereckgelenksprengung, Bankart-Läsion, superior labrum anterior posterior(SLAP)-Lä- sion oder eine posteriore Labrumverletzung sind Fakten, die schon vor einer Gipsabnahme geklärt sein sollten. Zusätzlich ist es wichtig, den Einsatzzweck mit dem Arzt zu klären und mit dem Patienten zu besprechen, da die Konstruktion darauf abgestimmt werden sollte.

Nach dem Gipsabdruck in Funktionsstellung, bei dem alle knöchernen Strukturen gut angezeichnet sein müssen, kann eine Kohlefaserschale, die Brust, Schulter und Schulterblatt umschließt und über ein Multiaxialgelenk mit einer Oberarmschale verbunden ist, hergestellt werden. Bei der Anprobe ist es möglich, über straffe oder elastische Zugzügel, die in Umlenkschlaufen geführt werden, das Bewegungsausmaß im Sinne der Außen-/ Innenrotation sowie der Abduktion statisch oder dynamisch zu limitieren. Die 

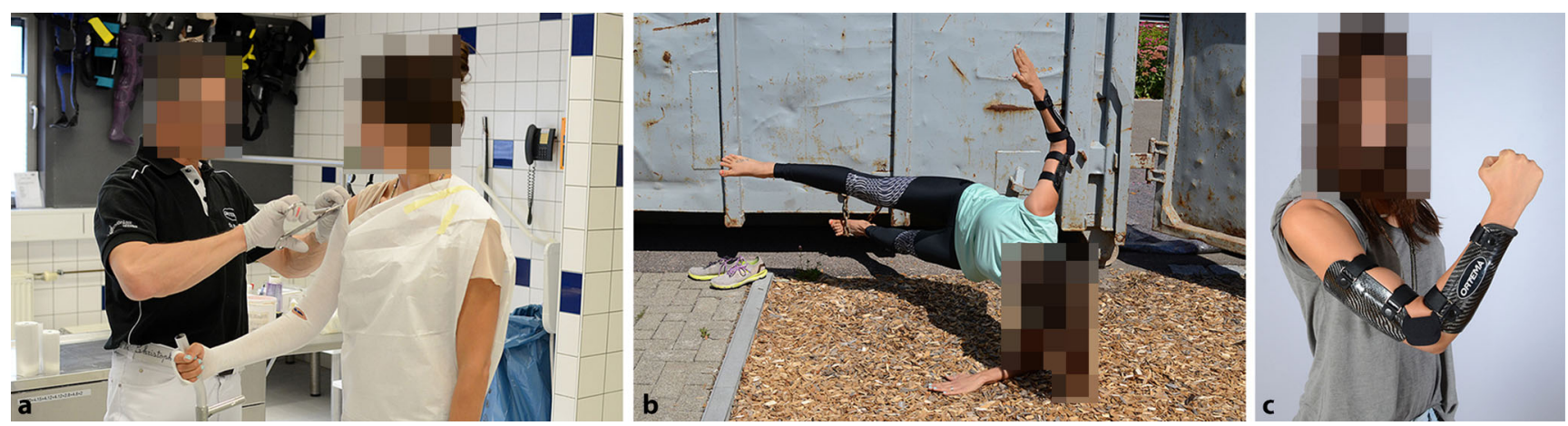

Abb. 6a-c $\Delta$ Snowboard-Olympiasiegerin verletzte sich bei einem Sturz das Ellenbogeninnenband. Die Orthese wird mit $10^{\circ}$ Extensionsanschlag gefertigt
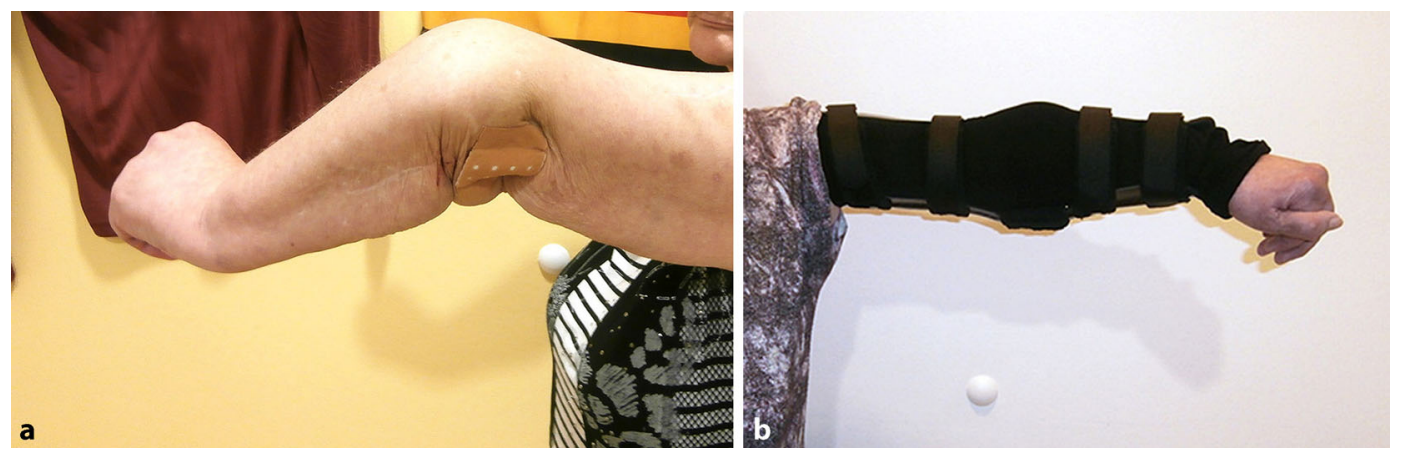

Abb. 7a,b $\triangleleft$ Ausgeprägte, völlig instabile Pseudoarthrose des Ellenbogens. Die Patientin lehnte eine Operation ab und wurde konservativ behandelt

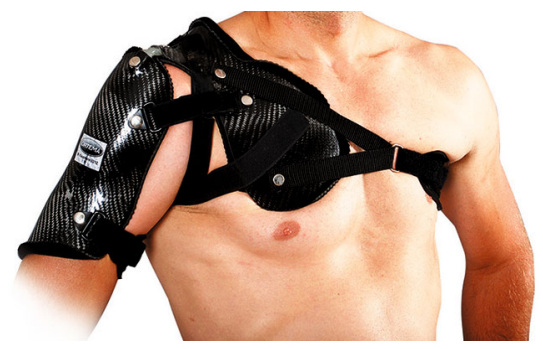

Abb. 8 ॥ Schulterversorgung des 14-fachen deutschen Downhill-Meisters mit individuell gefertigter Kohlefaserorthese nach mehrfacher Luxation

Schulterschale wird über einen Y-Zügel auf der Oberkörpergegenseite gut verankert, ein Verschluss unter der Axilla der betroffenen Seite fixiert die Orthese auf der Schulter auch bei der Bewegung (•Abb.8). Im Rahmen der Anprobe werden eine körperformschlüssige Ausarbeitung im Bereich der Klavikula und Scapula kontrolliert und Funktionstests bei Sportlern evtl. mit dem Sportgerät durchgeführt.

Die häufigste Ursache einer traumatischen Armplexuslähmung stellt die Traktion des Nervengeflechts nach Verkehrsunfällen, häufig Motorradunfälle, dar. Die Art der Nervenverletzungen ist wegen der unterschiedlichen Verletzungsmechanismen und Läsionsflächen groß, sie reicht von Dehnungen, Nervenwurzelausrissen aus dem Rückenmark über Nervenwurzelabrisse in unterschiedlicher Anzahl bis hin zu Läsionen des peripheren Armplexusgeflechtes [3]. Oftmals lässt sich direkt nach dem Trauma nicht eindeutig bestimmen, wie stark die Verletzung und die damit verbundenen Verletzungsausfälle sind. Es besteht nach Wochen und Monaten die Möglichkeit, dass sich die Nervenbahnen spontan erholen. Das ist v. a. nach Dehnungen und Zerrungen der Nerven möglich. Die Höhe der Schädigungen - oberhalb oder unterhalb des Schlüsselbeines - ist entscheidend für das Verletzungsmuster.

Sollte ein Patient mit Plexusparese zur Versorgung anstehen, muss genau definiert werden, welche Restfunktion im Bereich von Schulter, Bizeps/Trizeps, Ellenbogengelenk, Unterarm bzw. Hand und Finger vorliegt. Jegliche Restfunktion kann eine Bewegung unterstützen und sollte genau diagnostiziert werden. Zum Teil können Muskeltransferoperationen Funktionsverbesserungen erzeugen.
Die häufig schmerzhafte Subluxation des Humeruskopfes sollte möglichst bei der Konstruktion Berücksichtigung finden und vermieden werden.

Die unterschiedlichen Lähmungsmuster führen $\mathrm{zu}$ unterschiedlichen Muskelkraftgraden und Bewegungsausmaßen und sind häufig für die Atrophie des M. deltoideus und supraspinatus verantwortlich.

Da es sich bei der Klientel der Armplexusläsionspatienten häufig um Motorradfahrer, also eine aktive Patientengruppe, handelt, ist der Wunsch nach Aufnahme von Aktivitäten nach der Verletzung oftmals stark gegeben. Bei umfangreichen traumatischen Plexusläsionen ist die Einbeziehung der Schulter, des Oberarms, des Unterarms und der Hand in der Orthese angezeigt.

Immer wieder wird von den Betroffenen der Wunsch des Fahrradfahrens angegeben, da jedoch bei einer komplexen Läsion die vorhandene Trizepsund Bizepsfunktion schwach oder nicht vorhanden ist, wurde über eine mechanische Ellenbogenstabilisierung zur effektiven Lenkung, aber v. a. auch beim Bremsen des Fahrrads nachgedacht. So 


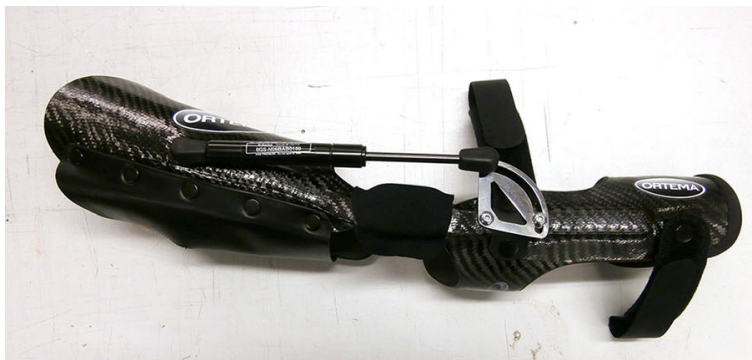

a

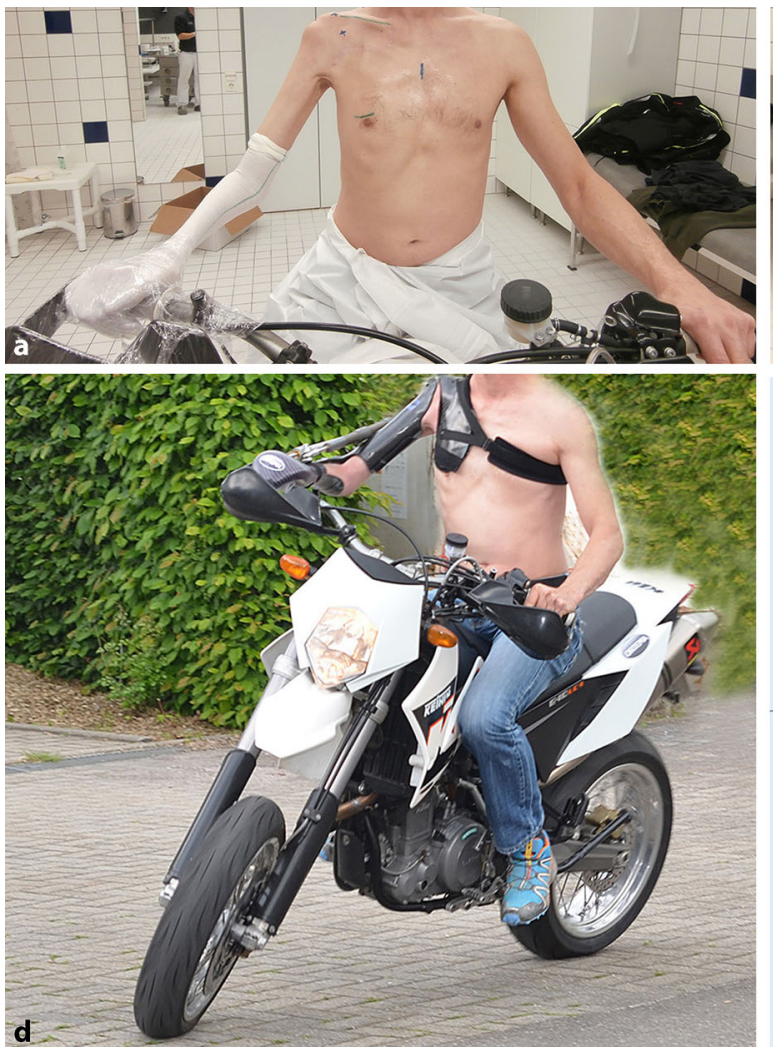

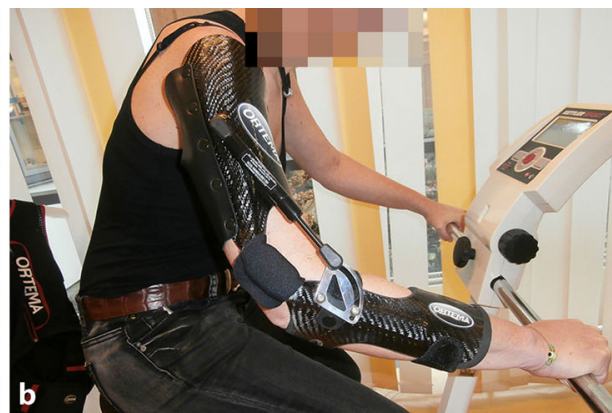

Abb. $9<$ a Über eine Radienplatte ist die optimale Anlenkung des Ellenbogengelenkdämpfers effektiv einstellbar. b Einsatz einer Plexusorthese bei einer Patientin mit Restfunktion der Hand
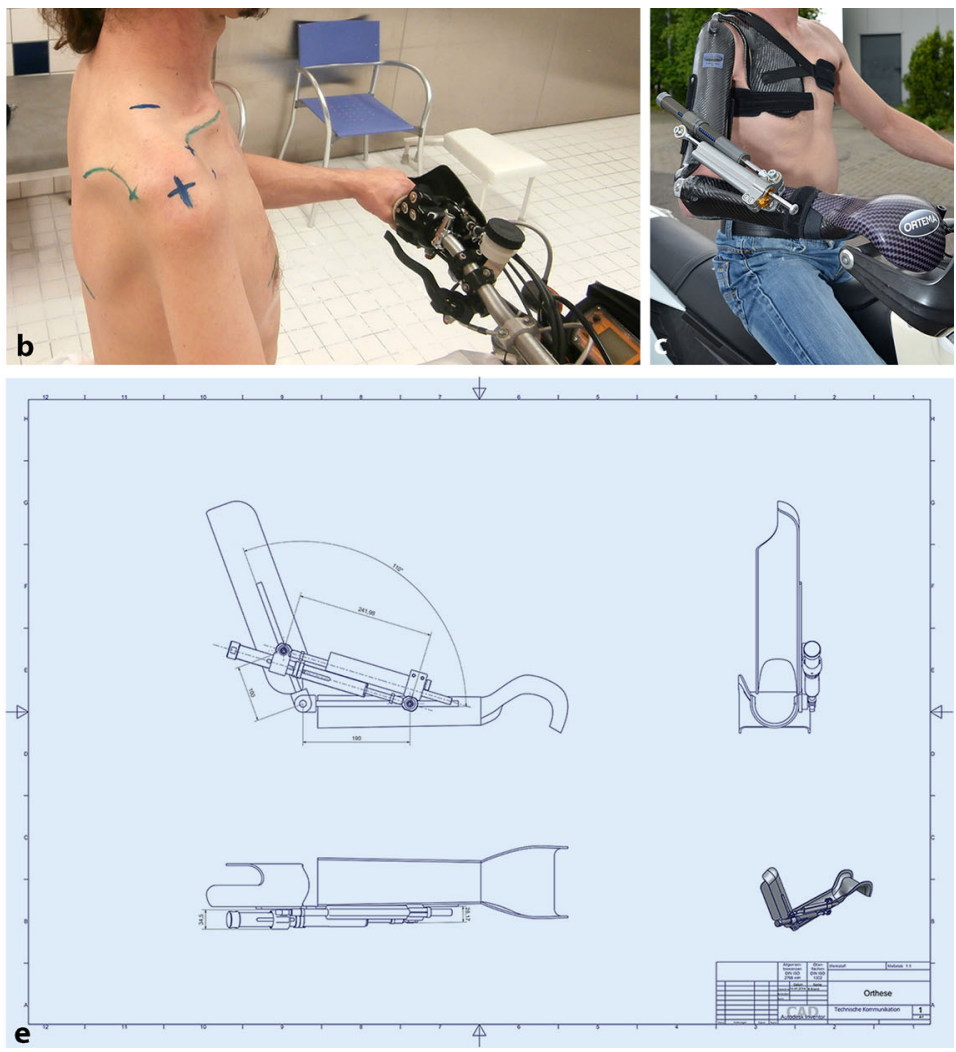

Abb. $10 \Delta$ a Gipsabdruck in individueller Funktionsstellung. b Korrekte Markierung der knöchernen Strukturen. c Plexusorthese mit Fixierung am Motorradlenker. d Motorradfahrer mit Orthese im Einsatz. e CAD-Konstruktionszeichnung

wurden nach Berechnung der Anlenkpunkte eines speziellen hydraulischen Stoßdämpfers zur Funktionsunterstützung des Ellenbogens Adapter in die Kohlefaserkonstruktion eingegossen, um den einstellbaren Dämpfer fixieren zu können (• Abb. 9).

Dieser muss auf das Gewicht und die Aktivitäten des Patienten ausgelegt sein und sollte möglichst mit der nicht geschädigten Hand der Körpergegenseite manuell schnell justierbar sein. Liegt keine Handfunktion vor, werden die Hand und die Langfinger in einer Art Lagerungsschale fixiert und über einen speziellen Kunststoffadapter am Lenker des Fahrrads adaptiert. Dieser kann sich im Sturzfall lösen. Nach umfangreichen Tests mit hydraulischen und pneumatischen Dämpfersystemen scheint der Einbau von Hydraulikdämpfern das Mittel der Wahl.

Mittlerweile werden auch bei Plexusparesen Orthesensysteme beim Motorradfahren eingesetzt (• Abb. 10). Hier sind die Beschleunigungs- und Verzögerungskräfte jedoch wesentlich höher, und das Dämpfungssystem muss dementsprechend größer dimensioniert und angepasst sein. Zum Teil werden auch 2 unterschiedlich wirkende Dämpfer- systeme montiert, die sich funktionell ergänzen.

\section{Ergebnisse}

Durch die intensive Beschäftigung mit Versorgungen von Patienten mit Verletzung der oberen Extremität konnten hilfreiche Erfahrungen gesammelt werden. Diese ermöglichen die Anfertigung komplexer Orthesen für den ganzen Arm unter Einbeziehung der Hand bei umfangreichen Plexusschädigungen.

Dies kann für den Benutzer bei Umschulungsmaßnahmen im Beruf, im Hobby, aber auch im Sport sehr hilf- 
reich sein. Die Erwartungshaltung und die Ansprüche der überwiegend aktiven Patienten steigen jedoch durch Funktionsverbesserung stark an und erwecken neue Aktivitätspotenziale.

\section{Fazit für die Praxis}

- Die orthetische Versorgung der oberen Extremität durch sehr dünne und leichte Konstruktionen ist sinnvoll.

- Orthesen sollten v. a. bei der Bewegung einen guten ortständigen Sitz mit optimaler Funktionalität vereinen.

- Bei Plexusverletzungen müssen geeignete hydraulische oder pneumatische einstellbare Dämpfersysteme zur Ellenbogenfunktionssteuerung gefunden werden, die eine möglichst individuelle Justierung für den Benutzer ermöglichen. Gerade die komplexe Orthesenversorgung scheint einen deutlichen Benefit für die Betroffenen zu haben, da sie die Aktivität und Leistungsfähigkeit des fast unbrauchbaren Arms deutlich steigert und verbessert.

- Da die Paresen typischerweise von Motorradfahrern bei Stürzen gegen harte Widerstände erlitten werden, haben wir es mit einer aktiven Patientenklientel zu tun.

- Diese nimmt durch ihr technisches Interesse jegliche Art an funktioneller Verbesserung gerne auf, um es in Beruf und Freizeit durch die Orthesenversorgung optimal einzusetzen.

\section{Korrespondenzadresse}

\section{H. Semsch}

OMM ORTEMA GmbH

Kurt-Lindemann-Weg 10, 71706 Markgröningen, Deutschland

info@ortema.de

\section{Einhaltung ethischer Richtlinien}

Interessenkonflikt. H. Semsch gibt an, dass kein Interessenkonflikt besteht.

Dieser Beitrag beinhaltet keine vom Autor durchgeführten Studien an Menschen oder Tieren.

Alle Patienten, die über Bildmaterial oder anderweitige Angaben innerhalb des Manuskripts zu identifizieren sind, haben hierzu ihre schriftliche Einwilligung gegeben. Im Falle von nicht mündigen Patienten liegt die Einwilligung eines Erziehungsberechtigten oder des gesetzlich bestellten Betreuers vor.

The supplement containing this article is not sponsored by industry.

\section{Literatur}

1. Marquaß B, Josten C (2008) Sportverletzungen am Ellenbogengelenk. OP-Journal 24:150-157

2. Hierner R, Berger A (2009) Verpflanzung des Musculus pectoralis major zur Wiederherstellung der Ellenbogenbeugung bei posttraumatischen Armplexusschäden. Oper Orthoptraumatol 2:129-140

3. Rührmann $O$ (2007) Zentrale und priphere Lähmungen. In: Wirth CJ, Mutschler W (Hrsg) Praxis der Orthopädie und Unfallchirurgie; Nerven; Erkrankungen. Thieme, Stuttgart, S316-326

4. Stöckle U (Hrsg) (2010) Ellenbogenchirurgie-, Standardverfahren, Tipps und Tricks. Elsevier, München

5. Letsch R, Schmit-Neuerburg KP (2001) Tscherner Unfallchirurgie. Bandverletzungen und Luxationen des Ellenbogengelenkes, Bd. 1., S67-76

6. Intrinsic-plus-Stellung aus Gelenktag_2013_ Brüche_Hand_und_Handgelenk.pdf von Dr.med. Daniel Vonier Leitender Oberarzt der Klinik für Plastische, Hand-und Rekonstruktive Chirurgie 